\title{
Role, Targets and Regulation of (de)nitrosylation in Malignancy
}

\author{
Salvatore Rizza ${ }^{1 *}$ and Giuseppe Filomeni ${ }^{1,2 *}$ \\ ${ }^{1}$ Redox Signaling and Oxidative Stress Research Group, Cell Stress and Survival Unit, Center for Autophagy, Recycling and \\ Disease, Danish Cancer Society Research Center, Copenhagen, Denmark, ${ }^{2}$ Department of Biology, University of Rome Tor \\ Vergata, Rome, Italy
}

Keywords: ADH5, GSNOR, nitric oxide, NOS, nitrosylation, cancer, FAK1, HIF-1 $\alpha$

\section{NITRIC OXIDE INVOLVEMENT IN CANCER}

Nitric oxide (NO) is a free radical that can target cellular biomolecules directly, or by means of the activity of its metabolites (RNS) generated upon reaction with transition metals (e.g., $\mathrm{NO}^{+}$), oxygen (e.g., $\mathrm{N}_{2} \mathrm{O}_{3}$ ), or superoxide $\left(\mathrm{ONOO}^{-}\right)$. For instance, it is well-documented that $\mathrm{NO}$ and $\mathrm{RNS}$ affect DNA integrity and mitochondrial physiology, this leading to genetic mutations (1) and damage to the mitochondrial respiratory chain $(2,3)$, respectively. Processes ranging from apoptosis, angiogenesis, immunity, and neuronal physiology, all show seemingly contradictory behavior in response to NO. Indeed, the relevance of the steady-state NO concentrations represents a key

\section{OPEN ACCESS}

Edited by:

Saverio Marchi,

University of Ferrara, Italy

Reviewed by:

Francesco Moccia

University of Pavia, Italy

*Correspondence:

Giuseppe Filomen giufil@cancer.dk

Salvatore Rizza

rizza@cancer.dk

Specialty section:

This article was submitted to Molecular and Cellular Oncology,

a section of the journal

Frontiers in Oncology

Received: 13 June 2018

Accepted: 02 August 2018

Published: 04 September 2018

Citation:

Rizza S and Filomeni G (2018) Role,

Targets and Regulation of

(de)nitrosylation in Malignancy.

Front. Oncol. 8:334.

doi: 10.3389/fonc.2018.00334 determinant of its biological function. In support to this assumption, it has been demonstrated that cGMP-mediated processes occur at the low $\mathrm{n} M$ range, whereas higher NO concentrations cause protein kinase B (PKB)/Akt phosphorylation; stabilization of hypoxia inducible factor (HIF)-1 $\alpha$; phosphorylation of $\mathrm{p} 53$ and, at the $\mu M$ range, they can generate detrimental conditions usually referred as to nitrosative stress (Figure 1). Likewise, in tumor biology, it is now commonly accepted that high NO concentrations mediate apoptosis and cancer growth inhibition, whereas (relatively) low concentrations usually promote tumor growth and proliferation, this supporting the nature of "doubled-edged sword" molecule for $\mathrm{NO}(4,5)$. This dichotomy originates from the observations that the inducible form of NO synthase (iNOS or NOS2) was implicated in the macrophage-mediated tumor killing process $(6,7)$ (Figure 1). NOS $2^{-/}$mice develop intestinal tumors (8), thereby substantiating the protective role of NOS2 within host defense mechanisms $(9,10)$. In accordance, a growing body of evidence pointed out that NO-releasing drugs can be toxic for cancer cells.

On the other hand, low rate of NO production can promote tumor growth rather than killing. In line with this assumption, the overexpression of NOS isoforms has been detected in a wide range of human tumors. In particular, NOS2 has been found to be upregulated in melanoma, estrogen receptor-negative $\left(\mathrm{ER}^{-}\right)$-breast cancer, as well as in pancreatic, cervical liver and ovarian cancers (10). Moreover, NOS2 seems to be involved in maintaining physiologically relevant levels of NO to sustain the progression phase of carcinogenesis; mainly it is required to promote angiogenesis and to enhance the ability of cancer cells to counteract nutrient paucity in solid tumors and to metastasize $(10,11)$. NOS2 is also overexpressed in glioma stem cells, and its activity is required for the expression of the cell cycle inhibitor cell division autoantigen-1 (CDA1), which sustains growth and tumorigenicity (12). NOS2 has been also found to be upregulated in hepatocellular carcinoma (HCC), and is often increased in the hepatocytes of patients with chronic hepatitis and alcoholic cirrhosis, conditions that predispose to HCC (13-15). Notwithstanding all these lines of 
evidence, investigations on $\mathrm{NOS} 2^{-/-}$mice, in spontaneous and fibrosis-associated models of HCC, reveal little effect of NOS2derived NO on hepatocarcinogenesis (16), meaning that other players are also involved.

\section{S-nitrosylation and Cancer}

Redox signal underlying both pro-survival and death pathways, is a molecular information transduced by means of reactive cysteine residues that can undergo $S$-hydroxylation $(\mathrm{SOH})$, upon reaction with ROS (i.e., $\mathrm{H}_{2} \mathrm{O}_{2}$ ) or $S$-nitrosylation (SNO), the posttranslational modification induced by $\mathrm{NO}$, which is now emerging to underlie NO bioactivity (17). In the presence of a sulfhydryl group in their close proximity, both these modifications can resolve in a more stable disulfide bridge (S-thiolation, SS) (18-20). Actually, it has been recently questioned whether $S$-nitrosylation-given its nature of instable posttranslational modification-is able to convey the NOmediated signal, or just acts as mere intermediate for disulfide bridge formation (21). Whatever is the end effector (if directly the SNO group or, indirectly, the SS adduct), the extent of $S$ nitrosylation is determined by a delicate balance between: (i) the rate of NO production, which is catalyzed by NOSs (22, 23 ), (ii) the activity of a recently discovered class of enzymes termed nitrosylases $(24,25)$, and (iii) the efficiency of SNO removal, that is mediated by denitrosylases. $S$-nitrosoglutathione reductase (GSNOR) represents the prototype of this class of oxidoreductases and, so far, the only denitrosylase able to completely reduce $\mathrm{NO}$ moiety, reason why it has been also termed GSNO terminase (26-28). Notwithstanding current literature offers still conflicting lines of evidence about the role of NOS/NO system in cancer biology, even less is known on the role played by GSNOR and denitrosylation. In this scenario, it has been reported that GSNOR-ablated (GSNOR-KO) mice show predilection to hepatocellular carcinoma (HCC) in association with $S$-nitrosylation and proteasomal degradation of the DNA damage repair enzyme $O^{6}$-alkylguanine-DNA alkyltransferase (AGT) (29). As a result, the repair of carcinogenic $O^{6}$ alkylguanines is significantly impaired with a consequence increase in tumorigenesis $(29,30)$. Analyses performed on human HCC patients showed a significant decrease of GSNOR protein levels and activity in the 50\% of cases (30), arguing for a functional link between GSNOR-dependent $S$-nitrosylation and HCC. Although this evidence supports a driving role for GSNOR and excessive S-nitrosylation in HCC ontogenesis, it is still unknown whether they are also implicated in the other phases of carcinogenesis, e.g., tumor promotion and progression (31).

In this regard, it has been published that GSNOR deficient HCC cells have a compromised mitochondrial electron transport chain characterized by the upregulation of succinate dehydrogenase $(S D H)$, likely as an adapting response to the general impairment of the mitochondrial respiratory machinery (32) derived from excessive nitrosative stress. The hyper-nitrosylation of the mitochondrial chaperone TNF receptor-associated protein 1 (Trap1) has been identified as the molecular event responsible for such a rearrangement and, in turn, for the enhanced sensitivity of GSNOR-downregulating HCC to SDH-targeting mitochondrial drugs (32). Nevertheless, it is worth to note that the mean size of GSNOR-deficient tumor xenografts is larger (approximately the double) than parental (GSNOR-proficient) HCC (32), suggesting that excessive $S$-nitrosylation arising from GSNOR loss, might promote tumor progression and growth in vivo. This hypothesis finds support in a recent study correlating GSNOR downregulation with HER2 ${ }^{+}$breast cancer resistance to trastuzumab and poor patient prognosis (33). Altogether, these pieces of evidence argue for a new role of GSNOR in malignancy and resistant phenotypes of breast cancer. However, no evidence about the molecular mechanisms underneath has been provided so far.

\section{Known and Supposed Targets of S-nitrosylation in Aggressive Cancer}

Based on what above reported, it is plausible that impairments of denitrosylation capacity (e.g., upon GSNOR deficiency) modulates the function/activity of oncoproteins susceptible to $S$-nitrosylation. These defects, more specifically than a general increase of NO production (that could impact on a plethora of different targets and to a different extent) might account for a deregulated NO-signaling in carcinogenesis. This hypothesis is further sustained by a very recent study indicating that GSNORdeficiency (and excessive $S$-nitrosylation deriving from it), is a condition associated with aging $(34,35)$, which represents a major risk factor for cancer development. Actually, cancer might count as an aging disease, and shares with aging some common features (e.g., genomic instability, telomere shortening, oxidative stress, deregulation of nutrient sensing) that, indeed, characterize both disorders (36).

Besides those previously mentioned, and others well documented to play a role in apoptosis (e.g., p53, Bcl2, and Fas), many oncoproteins have been discovered in the last decades to undergo $S$-nitrosylation. The modification of oncoproteins and tumor suppressors by NO-independently on the effects induced, whether gain- or loss-of-function $(23,37)$-is emerging as a critical phenomenon associated with neoplastic transformation. Some of these NO-modified oncoproteins participate to signal transduction and are found mutated or modulated in cancer. Within this class of proteins in which $S$-nitrosylation has been identified as pro-oncogenic modification, we can list: (i) the GTPase Ras (nitrosylated at Cys118) (23, 38), which underlies cancer cell growth downstream of receptor-associated tyrosine kinases; (ii) the phosphatase and tensin homolog PTEN (nitrosylated at Cys83) (39), which regulates the levels of phosphatidyl inositole-3-phosphate/Aktdependent pathway; (iii) the protein kinase c-Src, which represents one of the master regulators of tumor proliferation, invasion and metastatic phenotype, and has been found to be nitrosylated at Cys498 (40) (Figure 1). Interestingly, this residue is conserved throughout the Src family of protein tyrosine kinases (SFKs) and, at least for other two members, i.e., Yes and Fyn, has been also reported to stimulate their activation (40). Focal adhesion kinase 1 (FAK1) is also comprised in the 

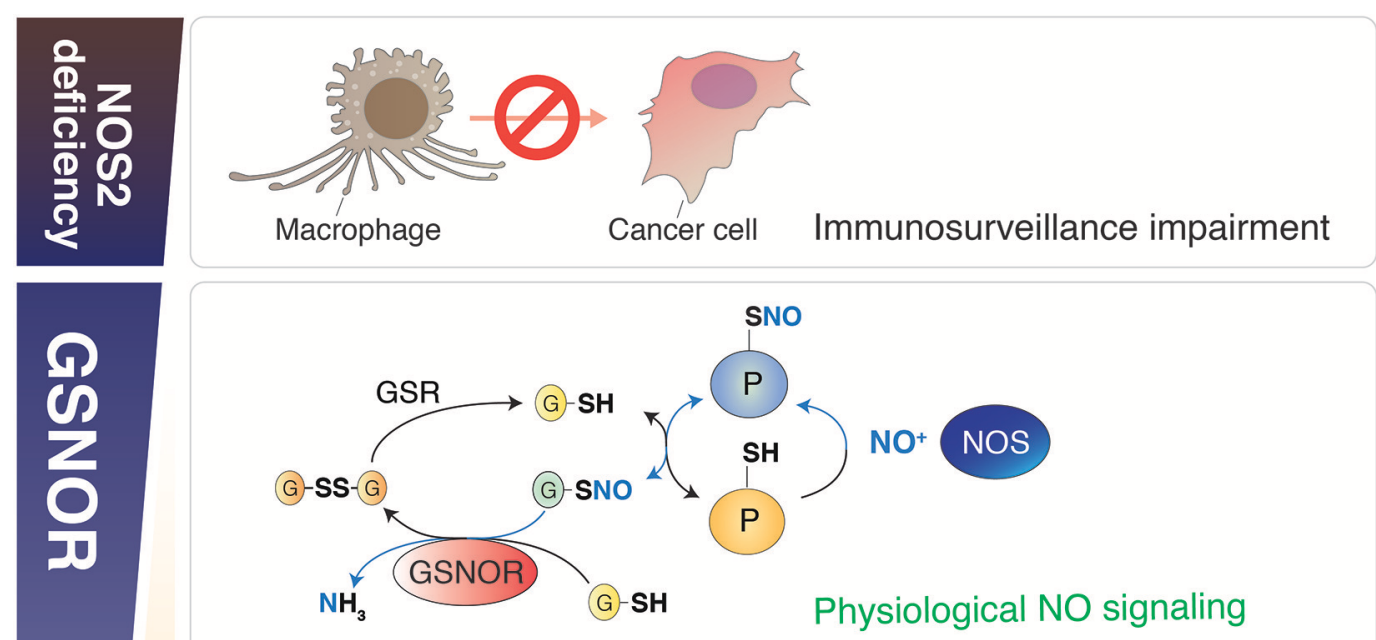

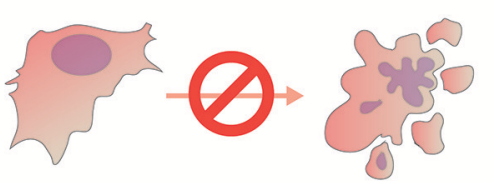

Apoptosis/anoikis resistance

Caspases

FAK1 (?)

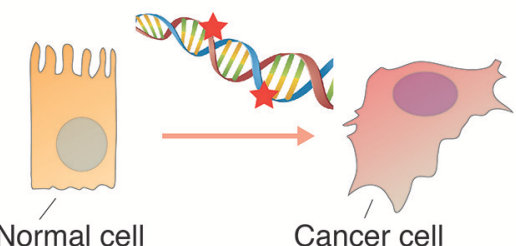

Genomic instability/mutagenesis AGT

Normal cell Cancer cell

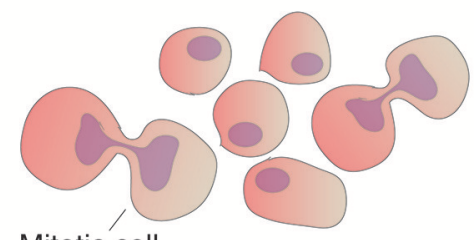

Mitotic cell

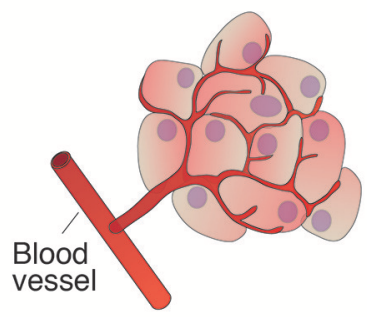

Hyperproliferation

PTEN/AKT

RAS

SrC

\section{Angiogenesis}

HIF-1a (?)

TRPS (?)
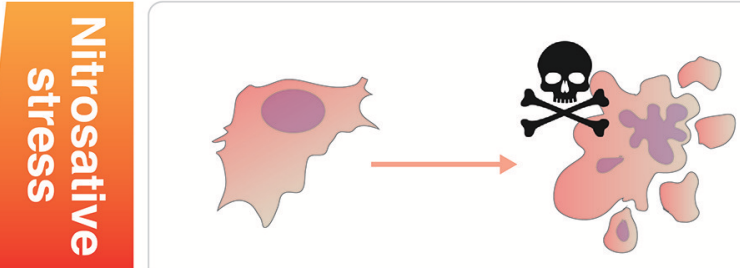

Apoptosis induction

FIGURE 1 | Roles of NO signaling and protein denitrosylation in cancer. Nitric oxide plays different roles in cancer biology depending on its concentration. GSNOR is the main cellular denitrosylase. Counteracting the effects induced by NOS, GSNOR finely modulates protein S-nitrosylation (second panel from the top), which is establishing as the main posttranslational modification underlying NO bioactivity. A disbalance in NO signaling can promote tumor induction, survival and progression. 
FIGURE 1 | NOS2 deficiency impairs the capability of macrophages to kill cancer cells (Top). Conversely, in conditions of normal (or induced) NOS activity, GSNOR decrease has been linked to many cancer hallmarks, such as: (i) apoptosis and anoikis resistance (due to caspases and, reasonably, FAK-1 S-nitrosylation); (ii) genomic instability (DNA repair impairment, due to AGT S-nitrosylation and degradation); (iii) cells hyperproliferation (via the NO-mediated activation of oncoproteins, such as AKT, RAS, and Src); (iv) angiogenesis (putatively regulated by HIF-1 $\alpha$ and TRPs S-nitrosylation). Extreme nitrosative stress conditions -induced, for instance, by NOS overexpression or by the use of NO-donors-activate cell death and are implemented (or physiologically activated in macrophages) to destroy cancer cells (Bottom). NO, nitric oxide; GSNOR, S-nitrosoglutathione reductase; NOS, nitric oxide synthase; FAK1, focal adhesion kinase 1; AGT, $0^{6}$-methylguanine-DNA methyltransferase; HIF- $1 \alpha$, hypoxia-inducible factor- $1 \alpha$; TRP, Transient receptor potential channel.

SFKs family. It is phosphorylated upon integrin engagement in a Src-dependent or independent (auto-phosphorylation) fashion, thus initiating multiple downstream signaling pathways responsible for aggressive and metastatic phenotype (e.g., resistance to anoikis and cell migration) (41). Similarly to Src, Yes and Fyn have been reported to act as FAK1-interacting kinases and to be involved in FAK1 activation as well $(42,43)$. Notwithstanding this tight relationship, triple-KO cells in which Src, Yes, and Fyn expression is suppressed (SYF cells), still show phospho-active levels of FAK1 upon treatment with NO donors (40). This unexpected evidence clearly indicates that $S$-nitrosylation of Src, Yes, and Fyn is dispensable for NO-driven phosphorylation of FAK1 and, interestingly, suggests that FAK1 might represent a direct target of $S$-nitrosylation (Figure 1), with this modification driving its oncogenic function.

Another oncoprotein, which has been identified to be crucial in cancer cell survival and growth, especially under low-oxygen tension (hypoxia), is HIF-1 $\alpha$. HIF-1 $\alpha$ deregulation has been deeply implicated in different aspects of cancer biology, such as angiogenesis, cell resistance, and tumor invasion (44-47). From a metabolic point of view, HIF- $1 \alpha$ aberrant activation underpins the so-called "Warburg effect": the preferential glycolytic consumption of glucose in cancer cells, which takes place also under normal oxygen tension. HIFl $\alpha$ has been found nitrosylated at Cys533 (48), with this being relevant in stroke and cardiovascular disease. However, if S-nitrosylation might somehow induce HIF1 $\alpha$ oncogenic activity still remains neglected (Figure 1) and would deserve to be investigated in the future.

Among the various classes of proteins that have been identified in the last decades as being activated by $S$-nitrosylation, the transient receptor potential (TRP) ion channels (49), which represent a huge family of proteins underpinning, among others, warm, taste, and pain sensory transduction, are worth to be mentioned. Besides their well-documented role in the nervous system as mediators of sensations, in the last years it is emerging that many TRPs, such as those belonging to the "melastatin" (TRPM), "vanilloid" (TRPV), and "ankyrin" (TRPA) subfamilies, are overexpressed in many cancer types, this being pivotal for calcium signaling-dependent control of tumor-promoting processes, e.g., vascularization and metastasis $(50,51)$. In particular, it has been proposed that, by modulating intracellular $\mathrm{Ca}^{2+}$ concentrations, TRPs are deeply involved in tumor initiation, progression and resistance (52). In this context, it has been very recently found out that TRPA1 is upregulated in breast and lung cancer downstream of the activation of $\mathrm{Nrf2}$, the master regulator of antioxidant response, this conferring non-canonical resistance to tumor cells against oxidative stress and ROS-producing chemotherapeutics (53). Many other observations argue for TRPs inhibition being a promising tool to eradicate cancer (54-56). However, notwithstanding the evidence that $S$-nitrosylation interferes with TRPs activity and calcium signaling, to date there's still no indication supporting a direct involvement of TRPs $S$ nitrosylation in carcinogenesis (Figure 1). Mostly, there's still no study aimed at understanding whether TRPs targeting on nitrosylable cysteines might represent a novel line of intervention in cancer treatment.

\section{Putative Mechanisms That Affect Denitrosylation in Cancer}

The above reported evidence points out that defects in GSNOR expression and denitrosylation are pivotal for sustaining the tumorigenic effects of $\mathrm{NO}$, namely, its role in the progression phase of cancer (31). A recent report on the epigenetic regulation of GSNOR might be of help to understand how this condition can be established in cancer. In particular, it has been demonstrated that GSNOR expression is controlled by the activity of the demethylase Ten-eleven translocation protein 1 (Tet1), a member of the 2-oxoglutarate-dependent dioxygenases that regulates transcription by removing methyl groups from $\mathrm{CpG}$ islands located in the promoter regions of genes (34). Remarkably, Tet1 expression has been found to be reduced in a wide range of solid cancers, such as melanoma, prostate, lung, and liver tumors $(57,58)$ - where also GSNOR mRNA seems to be downregulated-and to correlate with advanced cancer stage, nodal metastases, and poor survival rate in breast cancer patients (59). These lines of evidence suggest that GSNOR might be epigenetically downregulated in aggressive cancer as a consequence of Tet1 reduction, thus providing a new link between epigenetics and redox signaling. This hypothesis can be even extended to further mechanisms of epigenetic regulation. Indeed, given the complex structure of GSNOR mRNA, it has been proposed that GSNOR expression might be also regulated via microRNAs (miRs) (27). However, no putative miRs, able to target GSNOR transcript, has been so far identified to be upregulated in cancer, or hypothesized acting as additional modulators of $S$ nitrosylation.

\section{CONCLUSION}

The role of GSNOR-mediated denitrosylation in carcinogenesis has been capturing the interest of many researchers working on cancer biology, as many lines of evidence indicate that this 
process is frequently deregulated in cancer cells. In this article, we have tried to summarize what has been discovered in the last years and provide some hints on possible aspects that are still overlooked. Understanding how GSNOR expression is deregulated in may cancer histotypes, as well as the mechanisms underlying the modification of new protein targets involved in cancer resistance and aggressiveness, are, indeed, issues that deserve to be investigated in the future, since they could set the stage for new anticancer approaches interfering with the redox adaptation distinctive of many cancer cells.

\section{REFERENCES}

1. Dizdaroglu M, Jaruga P. Mechanisms of free radical-induced damage to DNA. Free Radic Res. (2012) 46:382-419. doi: 10.3109/10715762.2011.653969

2. Boveris A, Costa LE, Poderoso JJ, Carreras MC, Cadenas E. Regulation of mitochondrial respiration by oxygen and nitric oxide. Ann NY Acad Sci. (2006) 899:121-35. doi: 10.1111/j.1749-6632.2000.tb06181.x

3. Brown GC, Borutaite V. Nitric oxide inhibition of mitochondrial respiration and its role in cell death. Free Radic Biol Med. (2002) 33:1440-50. doi: 10.1016/S0891-5849(02)01112-7

4. Burke AJ, Sullivan FJ, Giles FJ, Glynn SA. The yin and yang of nitric oxide in cancer progression. Carcinogenesis (2013) 34:503-12. doi: 10.1093/carcin/bgt034

5. Vanini F, Kashfi K, Nath N. The dual role of iNOS in cancer. Redox Biol. (2015) 6:334-43. doi: 10.1016/j.redox.2015.08.009

6. Marletta MA. Nitric oxide synthase: aspects concerning structure and catalysis. Cell (1994) 78:927-30. doi: 10.1016/0092-8674(94)90268-2

7. Bogdan C. Nitric oxide and the immune response. Nat Immunol. (2001) 2:907-16. doi: 10.1038/ni1001-907

8. Yerushalmi HF, Besselsen DG, Ignatenko NA, Blohm-Mangone KA, PadillaTorres JL, Stringer DE, et al. The role of NO synthases in arginine-dependent small intestinal and colonic carcinogenesis. Mol Carcinog. (2006) 45:93-105. doi: $10.1002 / \mathrm{mc} .20168$

9. Xu W, Liu LZ, Loizidou M, Ahmed M, Charles IG. The role of nitric oxide in cancer. Cell Res. (2002) 12:311-20. doi: 10.1038/sj.cr.7290133

10. Thomas DD, Wink DA. NOS2 as an emergent player in progression of cancer. Antioxid Redox Signal (2017) 26:963-5. doi: 10.1089/ars.2016.6835

11. Jadeski LC, Hum KO, Chakraborty C, Lala PK. Nitric oxide promotes murine mammary tumour growth and metastasis by stimulating tumour cell migration, invasiveness and angiogenesis. Int J Cancer (2000) 86:30-9. doi: 10.1002/(SICI)1097-0215(20000401)86:1\&lt;30::AID-IJC5\&gt;3.0.CO;2-I

12. Eyler CE, Wu Q, Yan K, MacSwords JM, Chandler-Militello D, Misuraca KL, et al. Glioma stem cell proliferation and tumor growth are promoted by nitric oxide synthase-2. Cell (2011) 146:53-66. doi: 10.1016/j.cell.2011.06.006

13. Majano PL, García-monzón C, López-cabrera M, Lara-pezzi E, Fernándezruiz E, García-iglesias $\mathrm{C}$, et al. Inducible Nitric oxide synthase expression in chronic viral Hepatitis. evidence for a virus-induced gene upregulation. J Clin Invest. (1998) 101:1343-52.

14. Kane JM, Shears LL, Hierholzer C, Ambs S, Billiar TR, Posner MC. Chronic hepatitis $\mathrm{C}$ virus infection in humans: induction of hepatic nitric oxide synthase and proposed mechanisms for carcinogenesis. J Surg Res. (1997) 69:321-4. doi: 10.1006/jsre.1997.5057

15. McNaughton L, Puttagunta L, Martinez-Cuesta MA, Kneteman N, Mayers I, Moqbel R, et al. Distribution of nitric oxide synthase in normal and cirrhotic human liver. Proc Natl Acad Sci USA. (2002) 99:17161-6. doi: 10.1073/pnas.0134112100

16. Tang CH, Wei W, Hanes MA, Liu L. Hepatocarcinogenesis driven by GSNOR deficiency is prevented by iNOS inhibition. Cancer Res. (2013) 73:2897-904. doi: 10.1158/0008-5472.CAN-12-3980

17. Stamler JS, Lamas S, Fang FC. Nitrosylation: the prototypic redox-based signaling mechanism. Cell (2001) 106:675-83. doi: 10.1016/S0092-8674(01)00495-0

\section{AUTHOR CONTRIBUTIONS}

GF conceived the paper. GF and SR wrote the paper. SR drew the figure.

\section{ACKNOWLEDGMENTS}

This work has been supported by Danish Cancer Society Grant KBVU R146-A9414, and Associazione Italiana per la Ricerca sul Cancro (AIRC) Grant IG20719.

18. Sengupta R, Holmgren A. Thioredoxin and thioredoxin reductase in relation to reversible $S$-Nitrosylation. Antioxid Redox Signal (2013) 18:259-69. doi: 10.1089/ars.2012.4716

19. Martínez-Ruiz A, Araújo IM, Izquierdo-Álvarez A, Hernansanz-Agustín P, Lamas S, Serrador JM. Specificity in S-Nitrosylation: a short-range mechanism for NO signaling? Antioxid Redox Signal (2013) 19:1220-35. doi: 10.1089/ars.2012.5066

20. Jones DP, Sies H. The Redox Code. Antioxid Redox Signal (2015) 23: 734-46. doi: 10.1089/ars.2015.6247

21. Wolhuter K, Whitwell HJ, Switzer CH, Burgoyne JR, Timms JF, Eaton P. Evidence against stable protein S-Nitrosylation as a Widespread mechanism of post-translational regulation. Mol Cell (2018) 69:438-50.e5. doi: 10.1016/j.molcel.2017.12.019

22. Hess DT, Stamler JS. Regulation by S-Nitrosylation of protein post-translational modification. J Biol Chem. (2012) 287:4411-8. doi: 10.1074/jbc.R111.285742

23. Hess DT, Matsumoto A, Kim S-OO, Marshall HE, Stamler JS. Protein SNitrosylation: purview and parameters. Nat Rev Mol Cell Biol. (2005) 6:15066. doi: $10.1038 / \mathrm{nrm} 1569$

24. Seth D, Hess DT, Hausladen A, Wang L, Wang Y, Stamler JS. A multiplex enzymatic machinery for cellular protein S-Nitrosylation. Mol Cell (2018) 69:451-64.e6. doi: 10.1016/j.molcel.2017.12.025

25. Jia J, Arif A, Terenzi F, Willard B, Plow EF, Hazen SL, et al. Target-selective protein S-Nitrosylation by sequence motif recognition. Cell (2014) 159:62334. doi: 10.1016/j.cell.2014.09.032

26. Liu L, Hausladen A, Zeng M, Que L, Heitman J, Stamler JS. A metabolic enzyme for S-nitrosothiol conserved from bacteria to humans. Nature (2001) 410:490-4. doi: 10.1038/35068596

27. Rizza S, Filomeni G. Chronicles of a reductase: biochemistry, genetics and physio-pathological role of GSNOR. Free Radic Biol Med. (2017) 110:19-30. doi: 10.1016/j.freeradbiomed.2017.05.014

28. Jensen DE, Belka GK, Du Bois GC. S-Nitrosoglutathione is a substrate for rat alcohol dehydrogenase class III isoenzyme. Biochem J. (1998) 331(Pt. 2):659-68.

29. Wei W, Yang Z, Tang CH, Liu L. Targeted deletion of GSNOR in hepatocytes of mice causes nitrosative inactivation of O6-alkylguanine-dna alkyltransferase and increased sensitivity to genotoxic diethylnitrosamine. Carcinogenesis (2011) 32:973-7. doi: 10.1093/carcin/bgr041

30. Wei W, Li B, Hanes MA, Kakar S, Chen X, Liu L, et al. S-Nitrosylation from GSNOR deficiency impairs DNA repair and promotes hepatocarcinogenesis. Sci Transl Med. (2010) 2:19ra13. doi: 10.1126/scitranslmed.3000328

31. Rizza S, Filomeni G. Tumor suppressor roles of the denitrosylase GSNOR. Crit Rev Oncog. (2016) 21:433-45. doi: 10.1615/CritRevOncog.2017021074

32. Rizza S, Montagna C, Cardaci S, Maiani E, Di Giacomo G, Sanchez-Quiles $\mathrm{V}$, et al. S-Nitrosylation of the mitochondrial chaperone TRAP1 sensitizes hepatocellular carcinoma cells to inhibitors of succinate dehydrogenase. Cancer Res. (2016) 76:4170-82. doi: 10.1158/0008-5472.CAN-15-2637

33. Cañas A, López-Sánchez LM, Peñarando J, Valverde A, Conde F, Hernández V, et al. Altered S-nitrosothiol homeostasis provides a survival advantage to breast cancer cells in HER2 tumors and reduces their sensitivity to trastuzumab. Biochim Biophys Acta (2016) 1862:601-10. doi: 10.1016/j.bbadis.2016.02.005 
34. Rizza S, Cardaci S, Montagna C, Di Giacomo G, De Zio D, Bordi M, et al. S -Nitrosylation drives cell senescence and aging in mammals by controlling mitochondrial dynamics and mitophagy. Proc Natl Acad Sci USA. (2018) 21:7-13. doi: 10.1073/pnas.1722452115

35. Rizza S, Filomeni G. Denitrosylate and live longer: how GSNOR links mitophagy to aging. Autophagy (2018) 20:1-3. doi: 10.1080/15548627.2018.1475818

36. Aunan JR, Cho WC, Søreide K. The biology of aging and cancer: a brief overview of shared and divergent molecular hallmarks. Aging Dis. (2017) 8:628-42. doi: 10.14336/AD.2017.0103

37. Wang Z. Protein S-Nitrosylation and cancer. Cancer Lett. (2012) 320:123-9. doi: 10.1016/j.canlet.2012.03.009

38. Heo J, Campbell SL. Mechanism of p21Ras S-NITROSYLATION and kinetics of nitric oxide-mediated guanine nucleotide exchange. Biochemistry (2004) 43:2314-22. doi: 10.1021/bi035275g

39. Numajiri N, Takasawa K, Nishiya T, Tanaka H, Ohno K, Hayakawa W, et al. On-off system for PI3-kinase-Akt signaling through S-Nitrosylation of phosphatase with sequence homology to tensin (PTEN). Proc Natl Acad Sci USA. (2011) 108:10349-54. doi: 10.1073/pnas.1103503108

40. Rahman MA, Senga T, Ito S, Hyodo T, Hasegawa $H$, Hamaguchi M. S-Nitrosylation at cysteine 498 of c-Src tyrosine kinase regulates nitric oxide-mediated cell invasion. J Biol Chem. (2010) 285:3806-14. doi: 10.1074/jbc.M109.059782

41. Sulzmaier FJ, Jean C, Schlaepfer DD. FAK in cancer: mechanistic findings and clinical applications. Nat Rev Cancer (2014) 14:598-610. doi: 10.1038/nrc3792

42. Hamamura K, Tsuji M, Hotta H, Ohkawa Y, Takahashi M, Shibuya $\mathrm{H}$, et al. Functional activation of Src family kinase yes protein is essential for the enhanced malignant properties of human melanoma cells expressing ganglioside GD3. J Biol Chem. (2011) 286:18526-37. doi: 10.1074/jbc.M110.164798

43. Sen B, Johnson FM. Regulation of Src family kinases in human cancers. $J$ Signal Transduct. (2011) 2011:1-14. doi: 10.1155/2011/865819

44. Unwith S, Zhao H, Hennah L, Ma D. The potential role of HIF on tumour progression and dissemination. Int J Cancer (2015) 136:2491-503. doi: 10.1002/ijc.28889

45. Semenza GL. HIF-1 mediates metabolic responses to intratumoral hypoxia and oncogenic mutations. J Clin Invest. (2013) 123:3664-71. doi: $10.1172 /$ JCI67230

46. Rankin EB, Giaccia A J. The role of hypoxia-inducible factors in tumorigenesis. Cell Death Differ. (2008) 15:678-85. doi: 10.1038/cdd. 2008.21

47. Wu L, Fu Z, Zhou S, Gong J, Liu CA, Qiao Z, et al. HIF-1 $\alpha$ and HIF$2 \alpha$ : Siblings in promoting angiogenesis of residual hepatocellular carcinoma after high-intensity focused ultrasound ablation. PLoS ONE (2014) 9:1-10. doi: 10.1371/journal.pone.0088913

48. Li F, Sonveaux P, Rabbani ZN, Liu S, Yan B, Huang Q, et al. Regulation of HIF- $1 \alpha$ Stability through S-Nitrosylation. Mol Cell (2007) 26:63-74. doi: 10.1016/j.molcel.2007.02.024
49. Yoshida T, Inoue R, Morii T, Takahashi N, Yamamoto S, Hara Y, et al. Nitric oxide activates TRP channels by cysteine S-Nitrosylation. Nat Chem Biol. (2006) 2:596-607. doi: 10.1038/nchembio821

50. Fels B, Bulk E, Petho Z, Schwab A. The role of TRP channels in the metastatic cascade. Pharmaceuticals (2018) 11:E48. doi: 10.3390/ph11020048

51. Pla AF, Gkika D. Emerging role of TRP channels in cell migration: from tumor vascularization to metastasis. Front Physiol. (2013) 4:311. doi: 10.3389/fphys.2013.00311

52. Fliniaux I, Germain E, Farfariello V, Prevarskaya N. TRPs and $\mathrm{Ca}^{2+}$ in cell death and survival. Cell Calcium (2018) 69:4-18. doi: 10.1016/j.ceca.2017.07.002

53. Reczek CR, Chandel NS. ROS promotes cancer cell survival through calcium signaling. Cancer Cell (2018) 33:949-51. doi: 10.1016/j.ccell.2018.05.010

54. Almasi S, Kennedy BE, El-Aghil M, Sterea AM, Gujar S, PartidaSánchez $S$, et al. TRPM2 channel-mediated regulation of autophagy maintains mitochondrial function and promotes gastric cancer cell survival via the JNK-signaling pathway. J Biol Chem. (2018) 293:3637-50. doi: 10.1074/jbc.M117.817635

55. Bao L, Chen S, Conrad K, Keefer K, Abraham T, Lee JP, et al. Depletion of the human ion channel TRPM2 in neuroblastoma demonstrates its key role in cell survival through modulation of mitochondrial reactive oxygen species and bioenergetics. J Biol Chem. (2016) 291:24449-64. doi: 10.1074/jbc.M116.747147

56. Singh AK, Saotome K, McGoldrick LL, Sobolevsky AI. Structural bases of TRP channel TRPV6 allosteric modulation by 2-APB. Nat Commun. (2018) 9:2465. doi: 10.1038/s41467-018-04828-y

57. Liu C, Liu L, Chen X, Shen J, Shan J, Xu Y, et al. Decrease of 5hydroxymethylcytosine is associated with progression of hepatocellular carcinoma through downregulation of TET1. PLoS ONE (2013) 8:e62828. doi: 10.1371/journal.pone.0062828

58. Yang H, Liu Y, Bai F, Zhang JY, Ma SH, Liu J, et al. Tumor development is associated with decrease of TET gene expression and 5-methylcytosine hydroxylation. Oncogene (2013) 32:663-9. doi: 10.1038/onc.2012.67

59. Hsu CH, Peng KL, Kang ML, Chen YR, Yang YC, Tsai CH, et al. TET1 suppresses cancer invasion by activating the tissue inhibitors of metalloproteinases. Cell Rep. (2012) 2:568-79. doi: 10.1016/j.celrep.2012.08.030

Conflict of Interest Statement: The authors declare that the research was conducted in the absence of any commercial or financial relationships that could be construed as a potential conflict of interest.

Copyright (c) 2018 Rizza and Filomeni. This is an open-access article distributed under the terms of the Creative Commons Attribution License (CC BY). The use, distribution or reproduction in other forums is permitted, provided the original author(s) and the copyright owner(s) are credited and that the original publication in this journal is cited, in accordance with accepted academic practice. No use, distribution or reproduction is permitted which does not comply with these terms. 